\title{
HIV-1 Unspliced RNA Expression Induces Innate Immune Activation in
}

\section{Macrophages}

Hisashi Akiyama ${ }^{1}$, Caitlin M. Miller ${ }^{2}$, Chelsea R. Ettinger ${ }^{1}$, Anna C. Belkina ${ }^{1,3}$, Jennifer

Snyder-Cappione ${ }^{1}$ and Suryaram Gummuluru ${ }^{1}$

${ }^{1}$ Department of Microbiology, ${ }^{2}$ Department of Pathology, ${ }^{3}$ Flow Cytometry Core Facility,

Boston University School of Medicine, Boston, MA 02118

${ }^{*}$ Corresponding author

Suryaram Gummuluru, Ph.D.

Department of Microbiology

Boston University School of Medicine

72 E. Concord St., R512

Boston, MA 02118

Ph: (617) 414-8075

Fax: (617) 638-4286

Email: rgummulu@bu.edu 


\section{ABSTRACT}

Low-levels of type I interferons (IFN-I) are thought to be a driving force for immune activation and T cell exhaustion in HIV-1 infected individuals on highly active antiretroviral therapy (HAART), though the causative mechanisms for persistent IFN-I signaling have remained unclear. Here, we show Rev-CRM1 dependent nuclear export and peripheral membrane association of intron-containing HIV-1 unspliced RNA (usRNA), independent of primary viral sequence or viral protein expression, is subject to sensing, and results in IFN-I-dependent pro-inflammatory responses in macrophages. Our findings suggest that persistent expression of HIV-1 usRNA in macrophages contributes to chronic immune activation and that use of HIV RNA expression inhibitors as adjunct therapy might abrogate aberrant inflammation and restore immune function in HIV-infected individuals on HAART. 


\section{INTRODUCTION}

A hallmark of HIV-1 infection in vivo is systemic chronic immune activation (1), which has been postulated to lead to HIV-associated non-AIDS complications (HANA) (2) and dysfunction of T cells (3). Despite long-term viral suppression by HAART and restoration of $\mathrm{CD} 4^{+} \mathrm{T}$ cell levels, immune activation and inflammation persist in the majority of treated HIV-infected individuals, and is associated with excess risk of mortality and morbidity. Many factors have been attributed to cause this aberrant immune activation in vivo, such as bacterial endotoxin or co-infections (4); however, a viral (HIV) etiology for the chronic inflammatory state has remained unclear. Persistent infection of myeloid cells, most likely tissue-resident macrophages, is postulated to contribute to chronic immune activation and HANAs (5-7), though molecular mechanisms of how HIV-1 replication activates macrophages remain poorly understood.

In this study, we report that expression and Rev-CRM1 dependent nuclear export of HIV-1 unspliced RNA (usRNA) activates host sensing mechanisms and production of type I interferon (IFN-I)-dependent pro-inflammatory responses in productively infected macrophages. Ability of cells to distinguish intron-containing HIV-1 usRNA from self mRNA was dependent on localization of non-self HIV usRNA at peripheral membrane sites. These findings suggest that novel therapeutic strategies that suppress viral usRNA expression and IFN-I signaling cascades in tissue macrophages might have immunologic and therapeutic benefit in HIV-1 infected individuals on HAART.

\section{RESULTS}




\section{Late step of HIV-1 replication in macrophages triggers immune activation}

HIV-1 infection of monocyte-derived macrophages (MDMs) results in induction of a myeloid cell specific ISG, CD169/Siglec1 (Fig 1A) (8) whose expression is dramatically up-regulated (5-fold) even upon low levels $(<0.3 \mathrm{U} / \mathrm{ml})$ of IFN- $\alpha$ exposure (data not shown). Pre-treatment of MDMs with HIV-1 fusion (maraviroc), reverse transcription (AZT) or integration (raltegravir) inhibitors that blocked establishment of virus infection

(Fig 1B), abrogated induction of ISGs, CD169 (Fig 1C) and IP-10 (CXCL10) (Fig 1D). Interestingly, inhibition of Tat-dependent transcription (flavopiridol), but not virus particle maturation (treatment with HIV-1 protease inhibitor, indinavir), in HIV-infected MDMs

(Fig 1B), blocked enhancement of CD169 expression (Fig 1C) and secretion of IP-10

(Fig 1D), suggesting that a post-transcriptional step in HIV-1 replication cycle activates MDMs. Furthermore, induction of IFN- $\beta$ mRNA expression in productively infected MDMs was detected only at 3 days post infection (Fig 1E), which was coincident with the up-regulation of CD169 (data not shown), further supporting the hypothesis that a late event in virus replication cycle induces IFN-I responses. Moreover, B18R, IFN-I neutralizing reagent, potently inhibited CD169 expression on MDMs (Fig 1G) and reduced IP-10 secretion (Fig $1 \mathrm{H}$ ), confirming the presence of low levels of bioactive IFN-I (below the detection limit of a highly sensitive bioassay (Fig 1I)) in HIV-infected MDM culture supernatants that had negligible impact on virus infection (spread) (Fig 1F). Collectively, these results suggest that host sensing of a late step of HIV-1 replication in MDMs induces IFN-I-dependent pro-inflammatory responses. 


\section{Rev-CRM1 dependent HIV-1 usRNA export is subject to host sensing mechanisms}

Though induction of pro-inflammatory responses have been described upon exposure to HIV-1 accessory or structural proteins in diverse cell types $(9,10)$, MDMs infected with Nef, Env, Vpu, Vpr, or Vif-deficient mutants (Fig 2A) displayed robust induction of CD169 and IP-10 expression (Fig 2B and C). Moreover, MDM infections with Gag p6 $(\Delta \mathrm{p} 6), \mathrm{NC}(\triangle \mathrm{NCp} 6), \mathrm{MA}(\triangle \mathrm{MA})$ truncation mutants, or Gag start codon mutant $\left(\mathrm{ATG}^{*}\right)$

(Fig 2D, E and H) that results in initiation of Gag translation from an in-frame internal ATG present at the N-terminus of CA (leading to expression of aberrant Gag) failed to abrogate CD169 or IP-10 induction (Fig 2F, G, I and J). Additionally, infection of MDMs with HIV-1 budding deficient mutant (PTAP-) (11), CA-mutants deficient for Gag-Gag interaction (12), and cyclophilin A (CyPA)-binding-deficient CA mutant G89V (Fig 2K, N and $\mathbf{Q}$ ) resulted in up-regulation of CD169 and IP-10 (Fig $\mathbf{2 L}, \mathbf{M}, \mathbf{O}, \mathbf{P}, \mathbf{R}$ and $\mathbf{S}$ ), suggesting that neither virion-release (13), cytoplasmic accumulation of higher-ordered Gag assembly intermediates nor CyPA-binding to de novo expressed Gag, a target of a "cryptic sensor" in myeloid dendritic cells (10), is required for MDM activation.

Furthermore, complete abrogation of Gag-pol expression ( $\Delta$ Gag-pol; Fig 2D) only modestly attenuated expression of CD169 and IP-10 (Fig 2U and V) in infected MDMs (Fig 2T), suggesting that structural and accessory proteins of HIV-1 do not encode immune-activation determinants.

To identify the post-transcriptional viral product that induces pro-inflammatory responses in MDM, we next assessed the effect of viral RNA on MDM activation. There are three classes of viral mRNAs (multiply-spliced, singly-spliced and unspliced) 
transcribed from the HIV-1 LTR (14) . Nuclear export of HIV-1 usRNA relies on RevRRE binding and a cellular mRNA transporter CRM1 $(15,16)$. Mutations that disrupt Rev binding to CRM1 (M10 mutant; Fig 3A) or delete RRE ( $\triangle$ RRE; Fig 3A) abrogate usRNA nuclear export and thus Gag expression, but not export and translation of multiply-spliced viral RNAs (i.e. GFP in place of nef; Fig 3A, B and C) $(15,16)$. Interestingly, infection of MDMs with the M10 mutant failed to up-regulate CD169 and IP-10 expression (Fig 3D and E). Insertion of the constitutive transporting element (CTE) from Mason-Pfizer monkey virus (MPMV) (17) within the pol open reading frame (orf) of the M10 mutant (M10-CTE) or the $\triangle \mathrm{RRE}$ mutant ( $\triangle \mathrm{RRE}-\mathrm{CTE})$ in the sense orientation, but not in the anti-sense orientation (M10-CTE-AS), rescued nuclear export of HIV-1 usRNA and Gag expression (Fig 3A and B). However, rescue of HIV-1 usRNA nuclear export by CTE-dependent pathway failed to induce CD169 or IP-10 expression (Fig 3D and E). Moreover, while Rev-dependent expression of a non-viral protein (TagRFP) from the intron-containing viral usRNA (Fig 3F and G) induced CD169 and IP-10 expression (Fig 3H and I), Rev-independent expression of intron-less non-viral protein (ZsGreen) (Fig 3F and J) failed to induce CD169 and IP-10 expression (Fig 3K and L). These findings suggest that engagement of divergent RNA nuclear export pathways such as those mediated by Rev-RRE and MPMV CTE might lead to altered exposure of viral usRNA to host cytoplasmic sensing machinery, and that host sensing of viral usRNA specifically exported via Rev-CRM1 dependent pathway is necessary for inducing pro-inflammatory responses in MDMs.

\section{Membrane targeting of HIV-1 usRNA is required for MDM activation}


HIV-1 usRNA exported via Rev-RRE or CTE dependent pathway results in distinct cytoplasmic distribution (18). We therefore tested the hypothesis that cytoplasmic localization of HIV-1 usRNA-associated ribonucleoprotein complexes (RNPs) specific to the Rev-RRE pathway dictates induction of pro-inflammatory signaling cascades. Since a subset of cytoplasmic HIV-1 usRNAs are trafficked with Gag to membrane-associated virus assembly sites for packaging into new virions $(12,14)$, we infected MDMs with a series of Gag-MA mutants to potentially modulate localization of Gag-containing HIV-1 usRNA-associated RNPs. We found that none of the well-characterized MA membrane targeting domain mutants or those that potentially alter Gag assembly structure or HIV usRNA-Gag association prevented MDM activation (Fig 4A, B and C). Membranetargeting function is well conserved amongst all retroviral MA proteins, though site of virus assembly varies amongst retroviruses (14). To putatively alter membrane association sites of HIV-1 usRNA, we next utilized a HIV-1/MLV chimeric virus, mMA12, which contains MA and p12 of MLV in place of HIV-1 MA (Fig 4D)(19). Interestingly, infection of MDMs with mMA12 attenuated CD169 and IP-10 expression in productively infected MDMs (Fig 4E, F and G). Finally, infection of MDMs with myristoylationdeficient MA mutant (G2A) (Fig 4H) that prevents stable membrane association of HIV1 Gag (12) attenuated viral usRNA-induced MDM activation (Fig $\mathbf{4 I}$ and $\mathbf{J}$ ). These results suggest that stable membrane association of viral usRNA is necessary for host sensing and induction of type I IFN-dependent pro-inflammatory signals in MDMs.

\section{DISCUSSION}


Whether HIV-1 infection of MDMs is subject to sensing has remained a matter of controversy. In this study, we show that a late step of HIV-1 replication, specifically, expression and Rev-CRM1 mediated nuclear export of viral usRNA and its peripheral membrane association in MDMs is subject to host sensing mechanisms and results in innate immune activation and induction of pro-inflammatory responses. Importantly, we hypothesize that Rev-CRM1 dependent nuclear export specifically "marks" HIV-1 usRNA for detection by cytoplasmic sensing mechanisms, since access to alternative CTE-dependent nuclear export pathway failed to induce pro-inflammatory responses. There seems to be no sequence specificity for the sensing mechanism since inclusion of non-viral sequences (TagRFP) in viral usRNA failed to abrogate production of proinflammatory responses. Alternatively, "tethering" of HIV-1 usRNA to distinct peripheral membrane sites appeared to be a critical determinant; altering membrane targeting (by MLV-MAp12) or preventing membrane association (HIV-1 MA-G2A mutant) of viral usRNA potently attenuated induction of pro-inflammatory responses. While the distinct trafficking pattern and composition of viral usRNA-containing RNPs has been hypothesized to confer translational advantage to HIV-1 mRNAs compared to bulk host mRNA (20), such differences might also subject viral usRNA to detection by host innate immune sensing mechanisms. We hypothesize that a yet-to-be-identified sensor that resides at or in the vicinity of HIV-1 Gag assembly site senses HIV-1 usRNA-containing RNP complexes and activates MDMs. Further study is warranted to identify viral RNP complexes and the host factor(s) that activate MDMs upon HIV-1 infection.

Tissue-resident macrophages are important cellular targets of HIV, estimated to compromise up to $4 \%$ of infected cells in vivo (21), and can remain persistently infected 
with HIV-1 even in individuals on HAART (5-7). HIV-1 usRNAs have been detected in $\mathrm{CD}^{+} \mathrm{T}$ cells and alveolar macrophages from patients on long-term $\operatorname{HAART}(22,23)$. While these RNAs may not lead to functional viral protein production, persistent expression of viral usRNA might trigger host sensing in MDMs, that we demonstrate is a potent stimulus of IFN-I dependent pro-inflammatory responses. It is possible that HIV-1 infection of tissue-resident macrophages induces low levels of IFN-I and bystander myeloid cell activation contributing to systemic immune activation. Unfortunately, current HAART regimen does not block virus RNA transcription in previously infected cells. Thus, the development and clinical application of inhibitors that decrease viral RNA expression such as Tat and Rev inhibitors $(24,25)$, especially in tissue macrophages, could alleviate systemic immune activation in HIV-infected individuals on HAART. 


\section{MATERIALS AND METHODS}

\section{Plasmids}

HIV-1 replication competent molecular clones, Lai and Lai/YU-2env, single-round reporter constructs, Lai $\Delta$ env and Lai $\Delta$ envGFP (GFP in place of the nef orf) have been described previously $(26,27)$. HIV-1 mutants, Lai $\Delta$ env $\Delta$ vpr, Lai/YU-2env and Lai/YU$2 \mathrm{env} \Delta \mathrm{vpu}$, have been previously reported $(26,28)$. To create Lai $\Delta$ envGFP $\Delta$ vif, a vifcontaining fragment from Lai $\Delta$ env-luc $\Delta$ vif (frame-shift insertions at the Ndel site in the vif orf) (29) was replaced into the corresponding region of Lai $\Delta$ envGFP.

Lai $\Delta$ envGFP $\Delta$ Gag-pol was created by inserting frame-shift mutations at the Clal site (nt 377 ) in the Env-expression construct which has a large deletion between $\mathrm{N}$-terminus of CA and IN (26) and the fragment containing the env and nef orfs was replaced with the corresponding portion of Lai $\Delta$ envGFP. The CA G89V R9-GFP mutant and its parental virus R9-GFP were generous gifts from Dr. Christopher R. Aiken (Vanderbilt University School of Medicine). The PTAP- mutant and its parental virus R9 were generously provided by Dr. Wesley I. Sundquist (University of Utah Health). The following Gag mutants were generous gifts from Dr. Jaisri Lingappa (University of Washington): VK181/182AA, K158A, P224A, RS100/102AA, EE75/76AA, $\Delta$ p6, $\triangle$ NCp6, G2A. mMA12 $\triangle$ envGFP was created by replacing the luciferase gene of MHIV mMA12 (a generous gift from Dr. Masahiro Yamashita, Aaron Diamond AIDS Research Center) with the GFP gene. Rev-deficient mutant (M10) was created by PCR-directed mutagenesis using the primer set (Table 1). To make RRE-deletion mutant ( $\triangle R R E)$, a Stul-Alel fragment in the env orf was deleted from Lai $\Delta$ envGFP. The CTE was PCRamplified using the primers (Table 1) and pCR4-CTE (a gift from Dr. Mario Chin, 
Addgene plasmid \# 36868) as template and three repeats of CTE were inserted into M10 or $\triangle$ RRE between the Bcll (nt 2011) and Nhel (nt 3467) sites in a sense or antisense direction. HIV-1 MA-deficient mutants, $\triangle \mathrm{MA}$ (lacking amino acid residues 6 to 125) and ATG $^{*}$, and the following Gag mutants were created by PCR mutagenesis (QuikChangell, Agilent) or overlapping PCR using the primers listed in Table 1: $\triangle \mathrm{MA}$, 12LE, 16EK, 21LS, 29/31KE, 30LE, 34VE, 69TR, 85YG, 98EV, 7A2T using Lai $\Delta$ envGFP as a backbone. To express TagRFP in place of HIV-1 MA, an Ncol site was PCR-generated into at nt 378 (41 nt downstream of Gag starting codon) of the

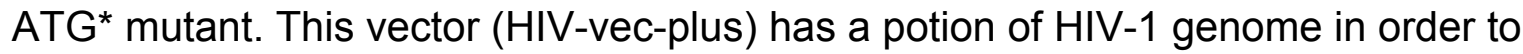
enhance chimeric viral RNA incorporation into virions (30). Then, an Aatll site was PCRgenerated just before the HIV-1 PR cleavage site into HIV-vec-plus (HIV-vec-plus-AatII) and PCR-amplified TagRFP (Evrogen) was inserted into HIV-1-vec-plus-AatlI. A lentivector expressing ZsGreen, pHAGE-ZsGreen, is a generous gift from Dr. Darrell Kotton (Boston University), and HIV-1 packaging plasmid psPAX2 and VSV-G expression constructs have been previously described (31).

\section{Cells}

Human monocyte-derived macrophages (MDMs) were derived from beads-isolated CD14 ${ }^{+}$peripheral blood monocytes (26) by culturing in RPMI1640 (Invitrogen) containing 10\% heat-inactivated human AB serum (Gemini Bio Products, Sigma or Corning) and recombinant human M-CSF (20 ng/ml; Peprotech) for 5-6 days. HEK293T, 293 ISRE-luc and TZM-bl have been described previously (28, 31, 32). 


\section{Viruses}

Replication competent viruses were derived from HEK293T cells via calcium phosphate transient transfection of HEK293T cells by calcium phosphate as described previously (31). Single-round-replication-competent viruses pseudotyped with VSV-G were generated from HEK293T cells via co-transfection of HIV-1 1 env constructs and VSV-G expression plasmid. To express HIV-1 mutants which by themselves cannot infect MDMs, a mutant plasmid was co-transfected with the packaging construct (psPAX2) and VSV-G expression vector into HEK293T (31). Virus-containing cell supernatants were harvested 2 days post-transfection, cleared of cell debris by centrifugation ( $300 \times \mathrm{g}$, $5 \mathrm{~min}$ ), passed through $0.45 \mu \mathrm{m}$ filters, and purified and concentrated by ultracentrifugation on a $20 \%$ sucrose cushion $\left(24,000 \mathrm{rpm}\right.$ and $4^{\circ} \mathrm{C}$ for 2 hours with a SW32Ti or SW28 rotor (Beckman Coulter)). The virus pellets were resuspended in PBS, aliquoted and stored at $-80{ }^{\circ} \mathrm{C}$ until use. The capsid content of HIV -1 was determined by a p24 ${ }^{\text {gag }}$ ELISA (31) and virus titer was measured on TZM-bl (33).

\section{Infection}

MDMs seeded one day before infection were spinoculated with viruses (1h at RT and $2,300 \mathrm{rpm}$ ) in the presence of polybrane (Milipore) at various moi (typically 0.5 to 2 ), cultured for $2-3$ hours at $37^{\circ} \mathrm{C}$, washed to remove unbound virus particles, and cultured for 3-6 days. Infection in MDMs was quantified by analyzing intracellular p24 ${ }^{\text {Gag }}$ or GFP expression by flow cytometry. In some experiments, MDMs were pretreated prior to infection or treated post infection with the following drugs/reagents: maraviroc $(\mathrm{NIH}$ AIDS Reagent Program), AZT (NIH AIDS Reagent Program), raltegravir (NIH AIDS 
Reagent Program or Selleckchem), flavopiridol (NIH AIDS Reagent Program), indinavir (NIH AIDS Reagent Program) and B18R (eBioscience).

\section{Immunoblot analysis}

To assess expression of $\mathrm{p} 24^{\text {gag }}$, cell lysates containing $20-30 \mu \mathrm{g}$ total protein were separated by SDS-PAGE, transferred to nitrocellulose membranes and the membranes were probed with a mouse anti-p24 antibody (p24-2, Dr. Michael H. Malim, NIH AIDS Reagent Program) followed by donkey anti-goat-IgG-IRDye 680 (Pierce). As loading controls, actin was probed using rabbit anti-actin (SIGMA) followed by a goat anti-rabbitIgG-IRDye 800CW (Pierce). Membranes were scanned with an Odessy scanner (LiCor).

\section{mRNA quantification}

Total mRNA was isolated from $1 \times 10^{6}$ cells using a kit (RNeasy kit, QIAGEN) and reverse-transcribed using oligo(dT) 20 primer (SuperscriptIII, Invitrogen). IFN- $\beta$ mRNA was quantified using Maxima SYBR Green (Thermo Scientific) and normalized to GAPDH mRNA by the $2^{-\Delta \Delta C}$ T method as described $(28,34)$. As a positive control for IFN$\beta$ mRNA expression, MDMs were treated with $1 \mu \mathrm{g} / \mathrm{ml}$ LPS (InvivoGen) for 2 hours.

\section{Cytokine quantification}

IP-10 in MDM culture supernatants was measured with a BD Human IP-10 ELISA Set (BD). Bioactive IFN-I was measured by a bioassay using 293 ISRE-luc cell line (28). 


\section{Flow cytometry}

CD169 on MDMs was stained with Alexa647-condjugated mouse anti-human CD169 antibody (BioLegend) and analyzed with BD LSRII (BD). Geo mean fluorescence intensity (MFI) was calculated and normalized to that of uninfected (mock) MDMs. In some experiments, intracellular p24 was stained as described (28) using FITC- or RD1condjucated mouse anti-p24 monoclonal antibody (KC57, Coulter). Data was analyzed with FlowJo software (FlowJo).

\section{Statistics}

All the statistics analysis was performed using GraphPad Prism 5. Two-tailed $p$ values were calculated using one-way ANOVA followed by the Tukey-Kramer post-test (symbols for $p$ values shown with a line) or the Dunnett's post-test (comparing to control (mock or wild type), symbols for $\mathrm{p}$ values shown on each column), or a paired t-test (comparing two samples, symbols for $p$ values shown with a line). Symbols represent, *: $p \leq 0.05,{ }^{* *}: p \leq 0.01,{ }^{* * *}: p \leq 0.001$, ns: not significant $(p>0.05)$. 


\section{Acknowledgments}

We thank Dr. Gregory A. Viglianti and Dr. Andrew J. Henderson for discussions and for critical reading of the manuscript. We thank Dr. Wesley I. Sundquist, Dr. Christopher R. Aiken, Dr. Jaisri Lingappa, Dr. Masahiro Yamashita and Dr. Darrell Kotton for generous gifts of reagents. We thank the BUMC Flow Cytometry Core and the Analytical Instrumentation Core for technical assistance. This work was supported by NIH grants Al064099 (SG), HD083111 (SG) and P30AI042853 (SG). 


\section{REFERENCES}

1. D. C. Douek, M. Roederer, R. A. Koup, Emerging concepts in the immunopathogenesis of AIDS. Annu Rev Med 60, 471-484 (2009).

2. M. Nasi, M. Pinti, S. De Biasi, L. Gibellini, D. Ferraro, C. Mussini, A. Cossarizza, Aging with HIV infection: a journey to the center of inflammAIDS, immunosenescence and neuroHIV. Immunol Lett 162, 329-333 (2014).

3. E. J. Wherry, T cell exhaustion. Nat Immunol 12, 492-499 (2011).

4. B. Taiwo, L. Barcena, R. Tressler, Understanding and controlling chronic immune activation in the HIV-infected patients suppressed on combination antiretroviral therapy. Curr HIVIAIDS Rep 10, 21-32 (2013).

5. J. H. Campbell, A. C. Hearps, G. E. Martin, K. C. Williams, S. M. Crowe, The importance of monocytes and macrophages in HIV pathogenesis, treatment, and cure. AIDS 28, 2175-2187 (2014).

6. J. B. Honeycutt, W. O. Thayer, C. E. Baker, R. M. Ribeiro, S. M. Lada, Y. Cao, R. A. Cleary, M. G. Hudgens, D. D. Richman, J. V. Garcia, HIV persistence in tissue macrophages of humanized myeloid-only mice during antiretroviral therapy. Nat Med, (2017).

7. M. Arainga, B. Edagwa, R. L. Mosley, L. Y. Poluektova, S. Gorantla, H. E. Gendelman, A mature macrophage is a principal HIV-1 cellular reservoir in humanized mice after treatment with long acting antiretroviral therapy. Retrovirology 14, 17 (2017).

8. P. R. Crocker, J. C. Paulson, A. Varki, Siglecs and their roles in the immune system. Nat Rev Immunol 7, 255-266 (2007).

9. N. C. Chen, A. T. Partridge, C. Sell, C. Torres, J. Martin-Garcia, Fate of microglia during HIV-1 infection: From activation to senescence? Glia 65, 431-446 (2017).

10. N. Manel, B. Hogstad, Y. Wang, D. E. Levy, D. Unutmaz, D. R. Littman, A cryptic sensor for HIV-1 activates antiviral innate immunity in dendritic cells. Nature 467, 214-217 (2010).

11. M. Huang, J. M. Orenstein, M. A. Martin, E. O. Freed, p6Gag is required for particle production from full-length human immunodeficiency virus type 1 molecular clones expressing protease. J Virol 69, 6810-6818 (1995).

12. B. A. Robinson, J. C. Reed, C. D. Geary, J. V. Swain, J. R. Lingappa, A temporospatial map that defines specific steps at which critical surfaces in the Gag MA and CA domains act during immature HIV-1 capsid assembly in cells. J Virol 88, 5718-5741 (2014).

13. R. P. Galao, A. Le Tortorec, S. Pickering, T. Kueck, S. J. Neil, Innate sensing of HIV-1 assembly by Tetherin induces NFkappaB-dependent proinflammatory responses. Cell Host Microbe 12, 633-644 (2012).

14. R. Swanstrom, J. W. Wills, in Retroviruses, J. M. Coffin, S. H. Hughes, H. E. Varmus, Eds. (Cold Spring Harbor (NY), 1997).

15. M. H. Malim, S. Bohnlein, J. Hauber, B. R. Cullen, Functional dissection of the HIV-1 Rev trans-activator--derivation of a trans-dominant repressor of Rev function. Cell 58, 205-214 (1989). 
16. D. S. Booth, Y. Cheng, A. D. Frankel, The export receptor Crm1 forms a dimer to promote nuclear export of HIV RNA. Elife 3, e04121 (2014).

17. H. Wodrich, A. Schambach, H. G. Krausslich, Multiple copies of the Mason-Pfizer monkey virus constitutive RNA transport element lead to enhanced HIV-1 Gag expression in a context-dependent manner. Nucleic Acids Res 28, 901-910 (2000).

18. G. M. Pocock, J. T. Becker, C. M. Swanson, P. Ahlquist, N. M. Sherer, HIV-1 and M-PMV RNA Nuclear Export Elements Program Viral Genomes for Distinct Cytoplasmic Trafficking Behaviors. PLoS Pathog 12, e1005565 (2016).

19. M. Yamashita, M. Emerman, Capsid is a dominant determinant of retrovirus infectivity in nondividing cells. J Virol 78, 5670-5678 (2004).

20. A. Sharma, A. Yilmaz, K. Marsh, A. Cochrane, K. Boris-Lawrie, Thriving under stress: selective translation of HIV-1 structural protein mRNA during Vprmediated impairment of elF4E translation activity. PLoS Pathog 8, e1002612 (2012).

21. S. A. Yukl, E. Sinclair, M. Somsouk, P. W. Hunt, L. Epling, M. Killian, V. Girling, P. Li, D. V. Havlir, S. G. Deeks, J. K. Wong, H. Hatano, A comparison of methods for measuring rectal HIV levels suggests that HIV DNA resides in cells other than CD4+ T cells, including myeloid cells. AIDS 28, 439-442 (2014).

22. H. Imamichi, R. L. Dewar, J. W. Adelsberger, C. A. Rehm, U. O'Doherty, E. E. Paxinos, A. S. Fauci, H. C. Lane, Defective HIV-1 proviruses produce novel protein-coding RNA species in HIV-infected patients on combination antiretroviral therapy. Proc Natl Acad Sci U S A 113, 8783-8788 (2016).

23. S. K. Cribbs, J. Lennox, A. M. Caliendo, L. A. Brown, D. M. Guidot, Healthy HIV1-infected individuals on highly active antiretroviral therapy harbor HIV-1 in their alveolar macrophages. AIDS Res Hum Retroviruses 31, 64-70 (2015).

24. N. Campos, R. Myburgh, A. Garcel, A. Vautrin, L. Lapasset, E. S. Nadal, F. Mahuteau-Betzer, R. Najman, P. Fornarelli, K. Tantale, E. Basyuk, M. Seveno, J. P. Venables, B. Pau, E. Bertrand, M. A. Wainberg, R. F. Speck, D. Scherrer, J. Tazi, Long lasting control of viral rebound with a new drug ABX464 targeting Rev - mediated viral RNA biogenesis. Retrovirology 12, 30 (2015).

25. G. Mousseau, M. A. Clementz, W. N. Bakeman, N. Nagarsheth, M. Cameron, J. Shi, P. Baran, R. Fromentin, N. Chomont, S. T. Valente, An analog of the natural steroidal alkaloid cortistatin A potently suppresses Tat-dependent HIV transcription. Cell Host Microbe 12, 97-108 (2012).

26. H. Akiyama, N. G. Ramirez, M. V. Gudheti, S. Gummuluru, CD169-mediated trafficking of HIV to plasma membrane invaginations in dendritic cells attenuates efficacy of anti-gp120 broadly neutralizing antibodies. PLoS Pathog 11, e1004751 (2015).

27. S. C. Hatch, J. Archer, S. Gummuluru, Glycosphingolipid composition of human immunodeficiency virus type 1 (HIV-1) particles is a crucial determinant for dendritic cell-mediated HIV-1 trans-infection. J Virol 83, 3496-3506 (2009).

28. C. M. Miller, H. Akiyama, L. M. Agosto, A. Emery, C. R. Ettinger, R. I. Swamstrom, A. J. Henderson, S. Gummuluru, Virion associated Vpr alleviates a post-integration block to HIV-1 infection of dendritic cells. J Virol, (2017). 
29. M. OhAinle, J. A. Kerns, H. S. Malik, M. Emerman, Adaptive evolution and antiviral activity of the conserved mammalian cytidine deaminase APOBEC $3 \mathrm{H}$. $J$ Virol 80, 3853-3862 (2006).

30. M. Reed, R. Mariani, L. Sheppard, K. Pekrun, N. R. Landau, N. W. Soong, Chimeric human immunodeficiency virus type 1 containing murine leukemia virus matrix assembles in murine cells. J Virol 76, 436-443 (2002).

31. W. B. Puryear, H. Akiyama, S. D. Geer, N. P. Ramirez, X. Yu, B. M. Reinhard, S. Gummuluru, Interferon-inducible mechanism of dendritic cell-mediated HIV-1 dissemination is dependent on Siglec-1/CD169. PLoS Pathog 9, e1003291 (2013).

32. H. Akiyama, C. Miller, H. V. Patel, S. C. Hatch, J. Archer, N. G. Ramirez, S. Gummuluru, Virus particle release from glycosphingolipid-enriched microdomains is essential for dendritic cell-mediated capture and transfer of HIV-1 and henipavirus. J Virol 88, 8813-8825 (2014).

33. C. A. Derdeyn, J. M. Decker, J. N. Sfakianos, X. Wu, W. A. O'Brien, L. Ratner, J. C. Kappes, G. M. Shaw, E. Hunter, Sensitivity of human immunodeficiency virus type 1 to the fusion inhibitor T-20 is modulated by coreceptor specificity defined by the V3 loop of gp120. J Virol 74, 8358-8367 (2000).

34. K. J. Livak, T. D. Schmittgen, Analysis of relative gene expression data using real-time quantitative PCR and the 2(-Delta Delta C(T)) Method. Methods 25, 402-408 (2001).

35. E. O. Freed, M. A. Martin, Virion incorporation of envelope glycoproteins with long but not short cytoplasmic tails is blocked by specific, single amino acid substitutions in the human immunodeficiency virus type 1 matrix. J Virol 69, 1984-1989 (1995).

36. A. Joshi, S. D. Ablan, F. Soheilian, K. Nagashima, E. O. Freed, Evidence that productive human immunodeficiency virus type 1 assembly can occur in an intracellular compartment. J Virol 83, 5375-5387 (2009).

37. N. M. Sherer, C. M. Swanson, S. Papaioannou, M. H. Malim, Matrix mediates the functional link between human immunodeficiency virus type 1 RNA nuclear export elements and the assembly competency of Gag in murine cells. J Virol 83, 8525-8535 (2009).

38. A. Ono, J. M. Orenstein, E. O. Freed, Role of the Gag matrix domain in targeting human immunodeficiency virus type 1 assembly. J Virol 74, 2855-2866 (2000).

39. E. O. Freed, M. A. Martin, Domains of the human immunodeficiency virus type 1 matrix and gp41 cytoplasmic tail required for envelope incorporation into virions. J Virol 70, 341-351 (1996).

40. P. R. Tedbury, S. D. Ablan, E. O. Freed, Global rescue of defects in HIV-1 envelope glycoprotein incorporation: implications for matrix structure. PLoS Pathog 9, e1003739 (2013).

41. E. O. Freed, J. M. Orenstein, A. J. Buckler-White, M. A. Martin, Single amino acid changes in the human immunodeficiency virus type 1 matrix protein block virus particle production. J Virol 68, 5311-5320 (1994).

42. V. Chukkapalli, S. J. Oh, A. Ono, Opposing mechanisms involving RNA and lipids regulate HIV-1 Gag membrane binding through the highly basic region of the matrix domain. Proc Natl Acad Sci U S A 107, 1600-1605 (2010). 


\section{FIGURE LEGENDS}

Fig 1. Late step of HIV-1 replication in macrophages triggers immune activation.

(A) Representative flow cytometry profiles of HIV-1-infected MDMs analyzed for CD169 expression and HIV-1 infection. (B) HIV-1 infection in MDMs in the presence or absence of inhibitors was measured by intracellular p24 ${ }^{\text {Gag }}$ staining. (C) CD169 expression (normalized to mock) and (D) IP-10 production in HIV-1-infected MDMs cultured in the presence of anti-HIV-1 drugs. (E) IFN- $\beta$ mRNA (normalized to untreated) expression in MDMs was quantified. (F) HIV-1 replication (intracellular p24 ${ }^{\mathrm{Gag}}$ ), (G) CD169 expression (normalized to mock) and (H) IP-10 production in HIV-1-infected MDMs cultured in the presence B18R. NT: untreated (DMSO). (I) IFN-I secretion from HIV-1-infected MDMs was measured by a bioassay with MDM supernatants harvested on 6 days post infection. The means \pm SEM are shown and each symbol represents an independent experiment. Two-tailed $p$ values: one-way ANOVA or paired t-test $(F),{ }^{* *}: p \leq 0.01,{ }^{* * *}: p$ $\leq 0.001$, ns: not significant.

\section{Fig 2. Structural and accessory proteins of HIV-1 do not encode immune- activation determinants.}

(A) HIV-1 infection (GFP expression or intracellular p24 ${ }^{\text {Gag }}$ ), (B) CD169 expression (normalized to mock), and (C) IP-10 production in MDMs infected with indicated HIV-1 mutants. m: mock. (D) Schematic demonstrations of HIV-1 Gag mutants. (E) HIV-1 infection (intracellular p24 ${ }^{\mathrm{Gag}}$ ), (F) CD169 expression (normalized to mock) and (G) IP10 production in MDMs infected with HIV-1 mutants lacking p6 or NC-p6. $(\mathrm{H}) \mathrm{HIV}-1$ infection (GFP expression), (I) CD169 expression (normalized to mock), and (J) IP-10 
production in MDMs infected with HIV-1 mutants lacking MA. (K) HIV-1 expression (intracellular p24 ${ }^{\mathrm{Gag}}$ ), (L) CD169 expression (normalized to mock), and (M) IP-10 production in MDMs transduced with the wild type (WT) or PTAP- mutant. (N) HIV-1 expression (intracellular p24 ${ }^{\mathrm{Gag}}$ ), (O) CD169 expression (normalized to mock), and (P) IP-10 production in MDMs transduced with the wild type (WT) or indicated CA mutants deficient for intra- or inter-hexamer formation. (Q) HIV-1 infection (GFP expression), (R) CD169 expression (normalized to mock), and (S) IP-10 production in MDMs transduced with the wild type (WT) or CyPA-binding-deficient mutant (G89V). (T) HIV-1 infection (GFP expression), (U) CD169 expression (normalized to mock), and (V) IP-10 production in MDMs infected with HIV-1 $\triangle$ Gag-pol mutant. The means \pm SEM are shown and each symbol represents an independent experiment. Two-tailed p values: one-way ANOVA or paired t-test $(\mathrm{K}, \mathrm{Q}$ and $\mathrm{T}),{ }^{*}: \mathrm{p} \leq 0.05,{ }^{* *}: \mathrm{p} \leq 0.01,{ }^{* * *}: \mathrm{p} \leq 0.001$, ns: not significant.

\section{Fig 3. Rev-CRM1 dependent HIV-1 usRNA export is required for sensing.}

(A) Schematic demonstration of HIV-1 Rev mutants and their Gag expression in infected MDMs. *: position of mutation, CTE: constitutive transport element, RRE: rev responsive element. (B) Quantification of Gag expression in infected MDMs (normalized to WT infection). (C) HIV-1 infection (GFP expression), (D) CD169 expression (normalized to mock), and (E) IP-10 production in MDMs infected with the HIV-1 Rev mutants. (F) Schematic demonstration of TagRFP and ZsGreen expression vectors. (G) HIV-1 infection (GFP expression), (H) CD169 expression (normalized to mock), and (I) IP-10 production in MDMs infected with the TagRFP mutant. (J) Transduction efficiency 
(GFP or ZsGreen expression), (K) CD169 expression (normalized to mock), and (L) IP10 production in MDMs transduced with the ZsGreen vector. The means \pm SEM are shown and each symbol represents an independent experiment. Two-tailed $p$ values: one-way ANOVA or paired t-test $(\mathrm{G}, \mathrm{J}),{ }^{*}: \mathrm{p} \leq 0.05,{ }^{* *}: \mathrm{p} \leq 0.01,{ }^{* * *}: \mathrm{p} \leq 0.001$, ns: not significant.

Fig 4. Membrane targeting of viral usRNA is required for MDM activation.

(A) Virus infection (GFP expression), (B) CD169 expression normalized to mock, and (C) IP-10 production in MDMs transduced with WT or indicated MA mutants lacking various MA functions (Table 1). (D) Schematic demonstration of MLV/HIV-1 chimera, mMA12. (E) Viral infection (intracellular p24 ${ }^{\text {Gag }}$ ), (F) normalized CD169 expression, and (G) IP-10 production in MDMs infected with WT or mMA12 viruses. (H) HIV-1 infection (intracellular p24 ${ }^{\mathrm{Gag}}$ ), (I) CD169 expression normalized to mock, and (J) IP-10 production in MDMs infected with WT or G2A mutant. The means \pm SEM are shown and each symbol represents an independent experiment. Two-tailed $p$ values: one-way ANOVA or paired t-test $(E, H),{ }^{* * *}: p \leq 0.001$, ns: not significant. 
bioRxiv preprint doi: https://doi.org/10.1101/174508; this version posted August 10,2017 . The copyright holder for this preprint (which was not certified by peer review) is the author/funder, who has granted bioRxiv a license to display the preprint in perpetuity. It is made available under aCC-BY-NC-ND 4.0 International license.

\section{Table 1}

\begin{tabular}{|c|c|c|c|}
\hline Plasmid & & Primer & Ref \\
\hline \multirow[t]{2}{*}{ M10 } & $\mathrm{F}$ & GCCTCTTCAGCTACCACCGGATCTGAGACTTACTCTTGATTGTAAC & (15) \\
\hline & $\mathrm{R}$ & GTTACAATCAAGAGTAAGTCTCAGATCCGGTGGTAGCTGAAGAGGC & \\
\hline \multirow[t]{2}{*}{ CTE } & $\mathrm{F}$ & TTTTTGGATCCACTATAGGGCGAATTGAATTTAGCG & $(17)$ \\
\hline & $\mathrm{R}$ & GGATAACAAT TTCACACAGG AAACAGCTAT GAC & \\
\hline \multirow[t]{2}{*}{ CTE AS } & $\mathrm{F}$ & TTTTTGCTAGCACTATAGGGCGAATTGAATTTAGCG & $(17)$ \\
\hline & $\mathrm{R}$ & TTTTTGGATCCCAGAATTAACCCTCACTAAAGGGAC & \\
\hline \multirow[t]{2}{*}{$\triangle M A$} & $\mathrm{~F}$ & TTTAAAGCTA GCCAGGTCAG CCAAAATTAC CCTATA & \\
\hline & $\mathrm{R}$ & AAATTTGCTA GCTCTCGCAC CCATCTCTCT CCTT & \\
\hline \multirow[t]{2}{*}{ ATG* } & $\mathrm{F}$ & GCTAGAAGGAGAGAGAAGGGTGCGAGAGCGT & \\
\hline & $\mathrm{R}$ & ACGCTCTCGCACCCTTCTCTCTCCTTCTAGC & \\
\hline \multirow[t]{2}{*}{ HIV-1 + Aatll } & $\mathrm{F}$ & AAAAAAGACGTCAGCCAAAATTACCCTATAGTGCAGAAC & \\
\hline & $\mathrm{R}$ & CTGGGTTCGCATTTTGGACC & \\
\hline \multirow[t]{2}{*}{ HIV-1-vec-plus } & $\mathrm{F}$ & AGAGGAGCTC TCTCGACG & $(30)$ \\
\hline & $\mathrm{R}$ & AAAAAACCATGGGATCTAATTCTCCCCCGCTTAATAC & \\
\hline \multirow[t]{2}{*}{ 12LE } & $\mathrm{F}$ & GTATTAAGCGGGGGAGAAGAAGATCGATGGGAAAAAATTCG & (35) \\
\hline & $\mathrm{R}$ & CGAATTTTTTCCCATCGATCTTCTTCTCCCCCGCTTAATAC & \\
\hline \multirow[t]{2}{*}{ 16EK } & $\mathrm{F}$ & GGGGAGAATTAGATCGATGGAAGAAAATTCGGTTAAGGCC & $(36)$ \\
\hline & $\mathrm{R}$ & GGCCTTAACCGAATTTTCTTCCATCGATCTAATTCTCCCC & \\
\hline \multirow[t]{2}{*}{ 21LS } & $\mathrm{F}$ & GATGGGAAAAAATTCGGTCAAGGCCAGGGGGAAAG & $(37)$ \\
\hline & $\mathrm{R}$ & СTTTCCCCCTGGCCTTGACCGAATTTTTTCCCATC & \\
\hline \multirow[t]{2}{*}{ 29/31KE } & $\mathrm{F}$ & GGGGGAAAGA AAAAATATGA ATTAGAACAT ATAGTATGGG C & (38) \\
\hline & $\mathrm{R}$ & GCCCATACTA TATGTTCTAA TTCATATTTT TTCTTTCCCC C & \\
\hline \multirow[t]{2}{*}{ 30LE } & $\mathrm{F}$ & GGCCAGGGGGAAAGAAAAAATATAAAGAAAAACATATAGTATGGGC & (35) \\
\hline & $\mathrm{R}$ & GCCCATACTATATGTTTTTCTTTATATTTTTTCTTTCCCCCTGGCC & \\
\hline \multirow[t]{2}{*}{ 34VE } & $\mathrm{F}$ & AAATTAAAACATATAGAATGGGCAAGCAGGGAGCTAGAAC & (39) \\
\hline & $\mathrm{R}$ & GTTCTAGCTCCCTGCTTGCCCATTCTATATGTTTTAATTT & \\
\hline \multirow[t]{2}{*}{ 69TR } & $\mathrm{F}$ & CTACAACCATCCCTTCAGAGAGGATCAGAAGAACTTAG & $(40)$ \\
\hline & $\mathrm{R}$ & CTAAGTTCTTCTGATCCTCTCTGAAGGGATGGTTGTAG & \\
\hline \multirow[t]{2}{*}{ 85YG } & $\mathrm{F}$ & ATACAGTAGCAACCCTCGGTTGTGTGCATCAAAGGATAG & $(41)$ \\
\hline & $\mathrm{R}$ & CTATCCTTTGATGCACACAACCGAGGGTTGCTACTGTAT & \\
\hline \multirow[t]{2}{*}{ 98EV } & $\mathrm{F}$ & GATAGAGATAAAAGACACCAAGGTTGCTTTAGACAAGATAGAGG & $(40)$ \\
\hline & $\mathrm{R}$ & ССTCTATCTTGTCTAAAGCAACCTTGGTGTCTTTTATCTCTATC & \\
\hline \multirow[t]{2}{*}{ 7A2T } & $\mathrm{F}$ & $\begin{array}{l}\text { GTTAGCGCCAGGGGGAGCGGCAGCATATACATTAACACATATAGTATGG } \\
\text { GCAAGCAGGGAGC }\end{array}$ & (42) \\
\hline & $\mathrm{R}$ & $\begin{array}{l}\text { CCGCTCCCCCTGGCGCTAACGCAATTGCTTCCCATGCATCTAATTCTCCC } \\
\text { CCGCTTAATACTGACG }\end{array}$ & \\
\hline
\end{tabular}




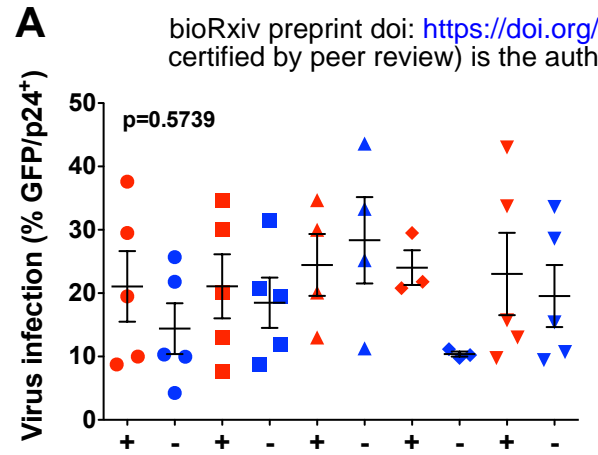

D

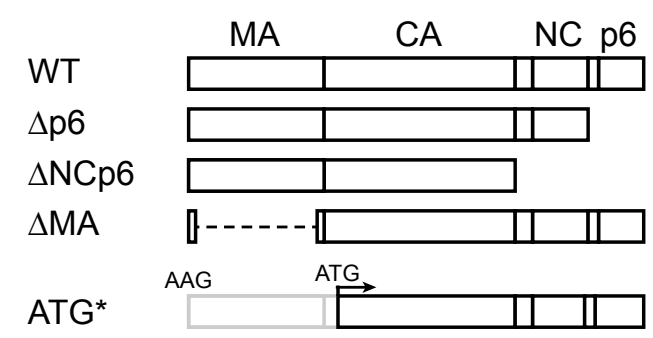

$\Delta$ Gag-pol $\square^{\text {TAA }}$

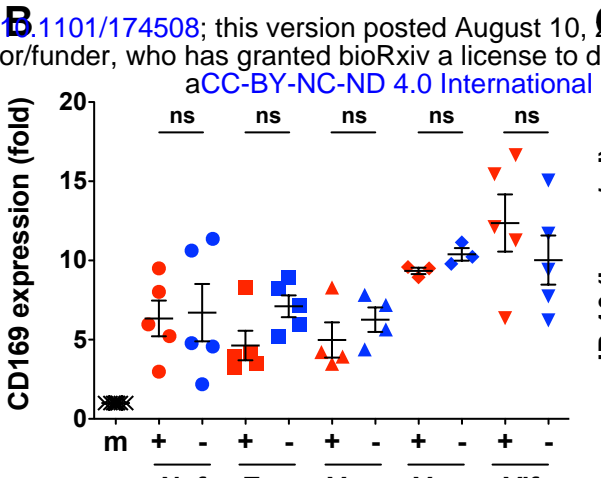

$\overline{\text { Nef }} \overline{\text { Env }} \overline{\text { Vpu }} \overline{\text { Vpr }} \overline{\text { Vif }}$
E

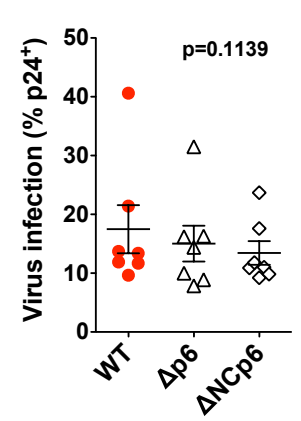

K

J
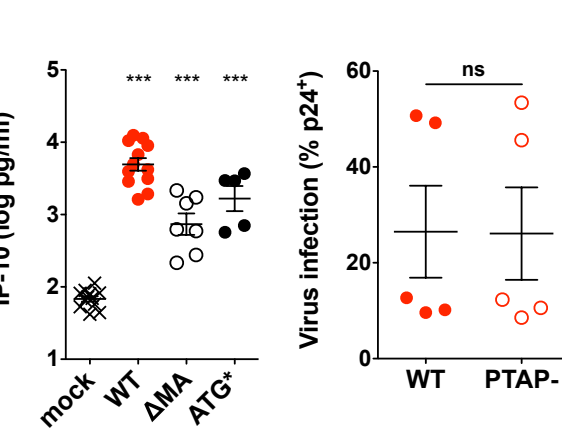

L

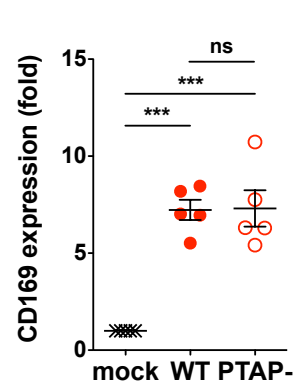

M
$\mathbf{F}$
G
$\mathbf{N}$

Q

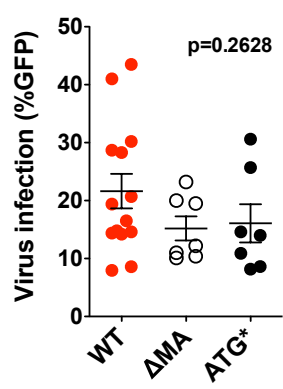

N

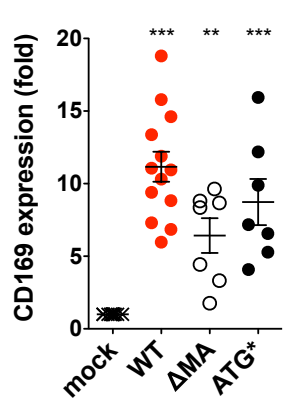

0
$P$

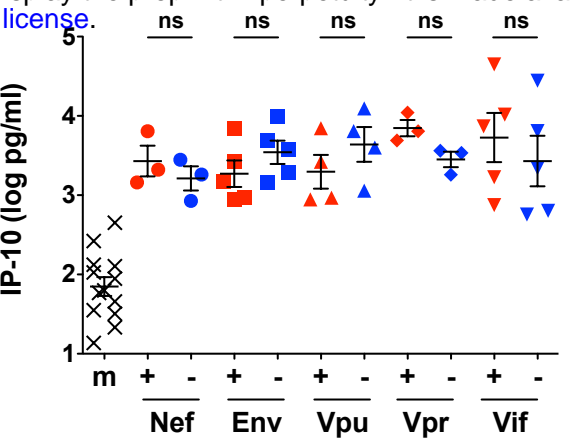


Akiyama et al., Fig 3

A

B C

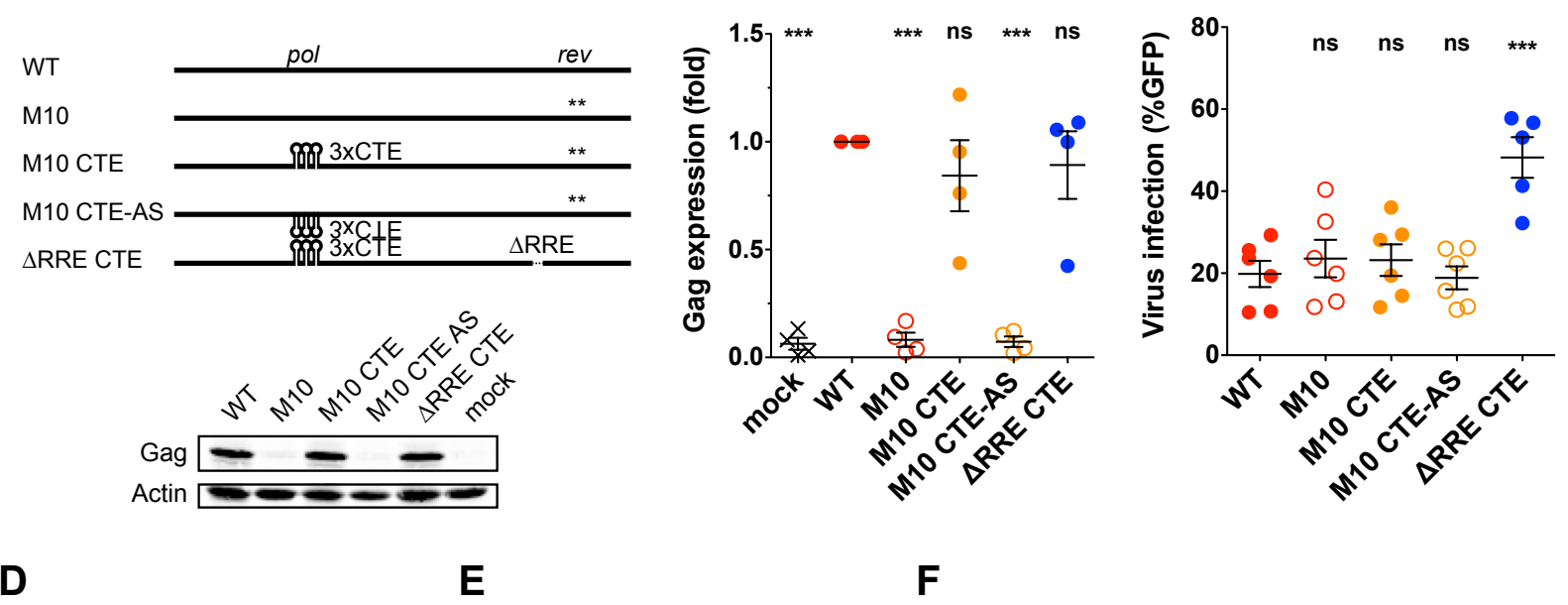

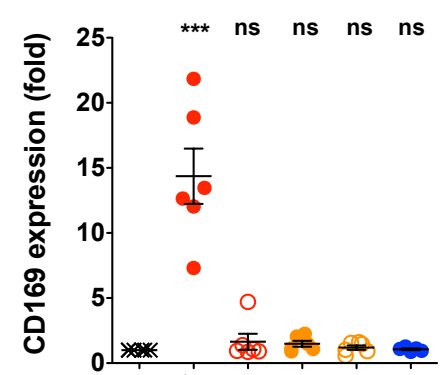

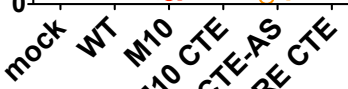

$\left.{ }^{5}\right] \quad * * *$ ns ns ns ns

NT

C

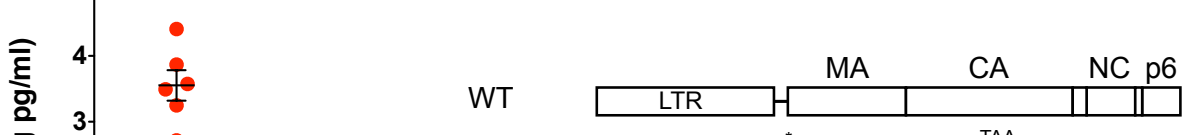

$\underbrace{0}_{0}$

TagRFP LTR TagRFP TA

ZsGreen EF1a $\quad$ ZsGreen

G

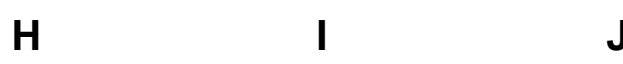

$\begin{array}{lll}\text { J } & \text { K } & \text { L }\end{array}$
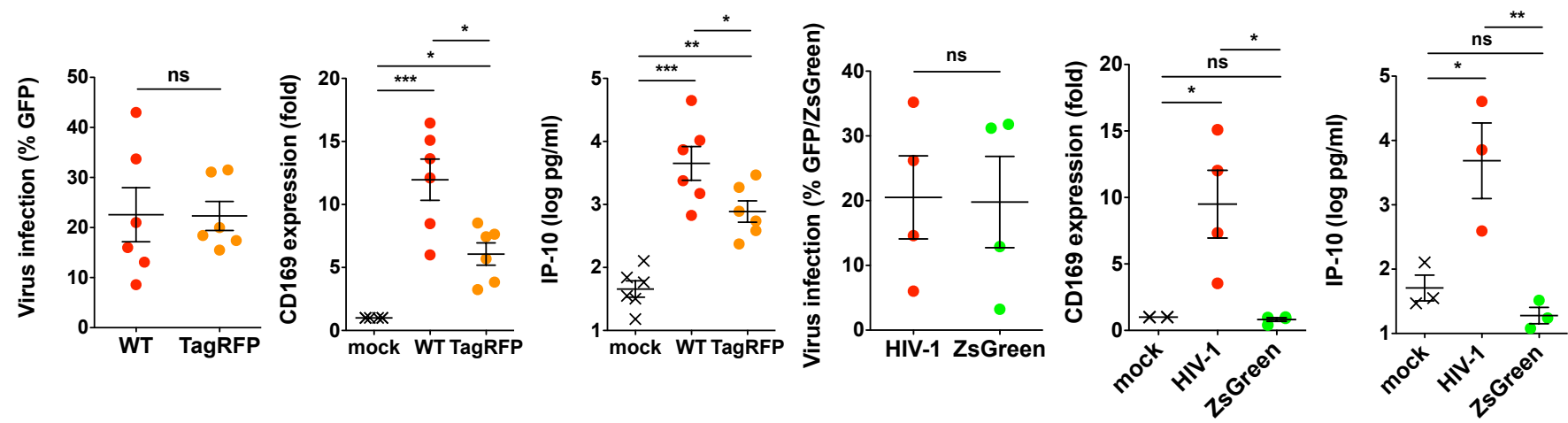
Akiyama et al., Fig 4

A

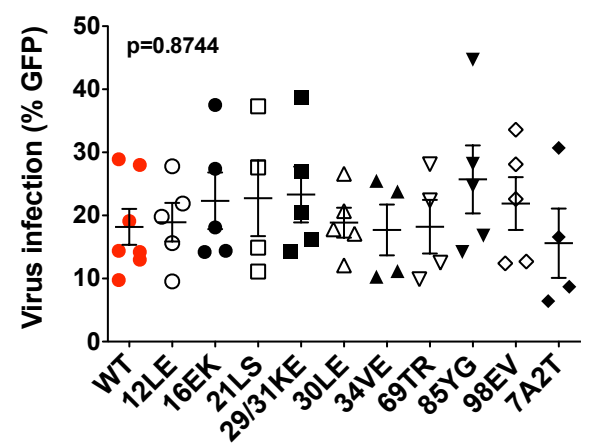

D

WT

mMA12

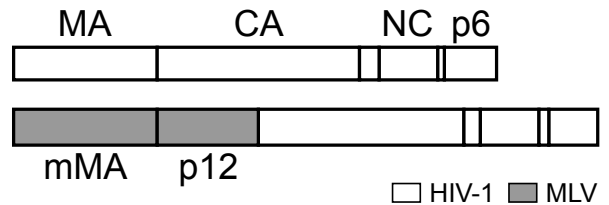

H

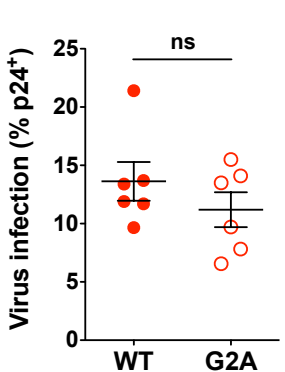

B

J

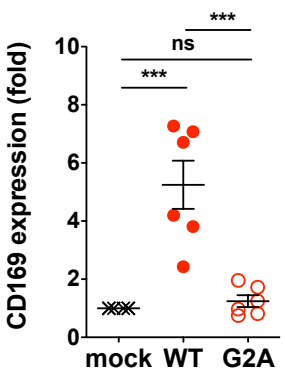

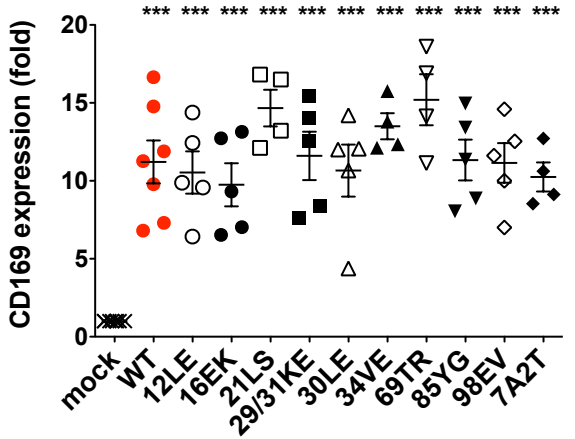

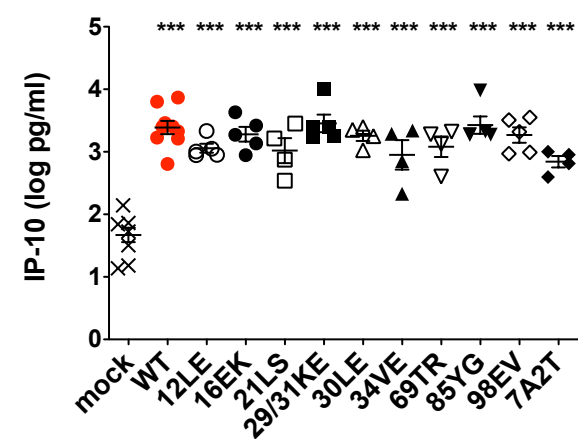

E

$\mathbf{F}$

G
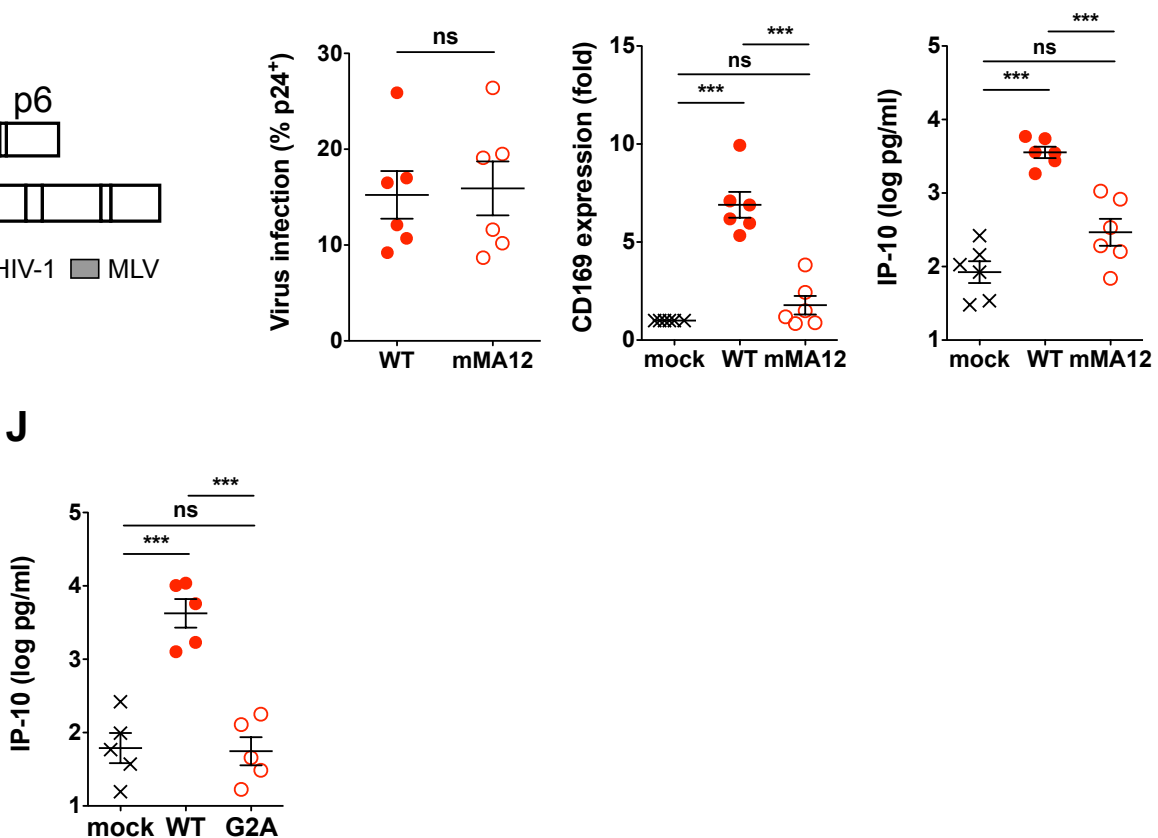